\title{
The Role of CD276 in Cancers
}

\section{OPEN ACCESS}

Edited by:

Andrew Zloza,

Rush University Medical Center,

United States

Reviewed by:

Ivana De La Serna,

University of Toledo Medical Center,

United States

Barbara Muz,

Washington University in St. Louis,

United States

*Correspondence:

Yiping Lu

luyiping_wch@126.com

Xianding Wang

xiandingwang@outlook.com

tORCID:

Shengzhuo Liu

0000-0001-8424-2549

Yiping Lu

0000-0003-1541-2879

Xianding Wang

0000-0002-5780-5622

${ }^{*}$ These authors have contributed equally to this work

Specialty section: This article was submitted to Cancer Molecular Targets and Therapeutics,

a section of the journal

Frontiers in Oncology

Received: 17 January 2021 Accepted: 02 March 2021

Published: 26 March 2021

Citation:

Liu S, Liang J, Liu Z, Zhang C, Wang Y, Watson AH, Zhou C, Zhang F, Wu K, Zhang F, Lu Y and Wang $X$ (2021) The

Role of CD276 in Cancers.

Front. Oncol. 11:654684. doi: 10.3389/fonc.2021.654684

\author{
Shengzhuo Liu ${ }^{1,2+\neq}$, Jiayu Liang ${ }^{1 \neq}$, Zhihong Liu ${ }^{1}$, Chi Zhang ${ }^{1}$, Yang Wang ${ }^{3}$, \\ Alice Helen Watson ${ }^{4}$, Chuan Zhou ${ }^{1}$, Fan Zhang ${ }^{1}$, Kan Wu ${ }^{1}$, Fuxun Zhang ${ }^{1}$, Yiping Lu ${ }^{1 *+}$ \\ and Xianding Wang ${ }^{1 *+}$
}

${ }^{1}$ Department of Urology, Institute of Urology, West China Hospital, Sichuan University, Chengdu, China, ${ }^{2}$ William Harvey Research Institute, Queen Mary University of London, London, United Kingdom, ${ }^{3}$ Laboratory of Aging Research and Cancer Drug Target, State Key Laboratory of Biotherapy, National Clinical Research Center for Geriatrics, West China Hospital, Sichuan University, Chengdu, China, ${ }^{4}$ Clinical Science and Services, Royal Veterinary College, University of London, London, United Kingdom

Objective: Aberrant expression of the immune checkpoint molecule, CD276, also known as $\mathrm{B} 7-\mathrm{H} 3$, is associated with tumorigenesis. In this review, we aim to comprehensively describe the role of CD276 in malignancies and its potential therapeutic effect.

Data Sources: Database including PubMed, EMbase, Cochrane Library, CNKI, and Clinical Trails.gov were searched for eligible studies and reviews. Study selection: Original studies and review articles on the topic of CD276 in tumors were retrieved.

Results: CD276 is an immune checkpoint molecule in the epithelial mesenchymal transition (EMT) pathway. In this review, we evaluated the available evidence on the expression and regulation of CD276. We also assessed the role of CD276 within the immune micro-environment, effect on tumor progression, and the potential therapeutic effect of CD276 targeted therapy for malignancies.

Conclusion: CD276 plays an essential role in cell proliferation, invasion, and migration in malignancies. Results from most recent studies indicate CD276 could be a promising therapeutic target for malignant tumors.

Keywords: CD276, B7-H3, therapeutic target, tumor, progression

\section{INTRODUCTION}

Immune Checkpoint Therapy (ICT) is a novel treatment for malignancy which shows great efficacy by enhancing anti-tumor immune response of $\mathrm{T}$ cells. The $\mathrm{B} 7$ family, which is important in regulating $\mathrm{T}$ cell immune response, has been highlighted for its potential therapeutic effect on cancers. B7 family members, including B7-H1 (PD-L1/CD274), B7-DC (PD-L2/CD273), B7-H2 (ICOSL), B7-H3 (CD276), B7-H4 (B7S1/B7x/Vtcn1), B7-H5 (VISTA/GI24/Dies/PD-1H), B7-H6 (NCR3LG1), B7-H7 (HHLA2), B7.1 (CD80), and B7.2 (CD86), could be categorized into (a) costimulatory, (b) co-inhibitory, or (c) mixed co-stimulatory and co-inhibitory subtype according to how they influence T cell activation (1).For example, PD-L1/B7-H1 is the inhibitory ligand of PD-1 death receptor and serves as an immune regulator by reducing T cell proliferation. B7-H3 (CD276), as a member of B7 family, was first introduced by Chapoval et al. in 2001 (2). Recent studies found a newly emergent immune checkpoint molecule, CD276, which serves as a T cell inhibitor to promote tumor proliferation and invasion, rather than CD276 acting as a T cell stimulatory molecule as previously described. Our study revealed that CD276 is an EMT pathway associated immune 
checkpoint. This review summarizes the available evidence to the expression of CD276 in tumors and its regulation mechanism, CD276 in immune micro-environment, effect on tumor progression, and its potential therapeutic effect for malignancies.

\section{EXPRESSION AND REGULATION OF CD276 IN TUMOR}

\section{Expression Levels of CD276 in Tumors}

CD276 mRNAs are commonly expressed at a relatively low level in most normal tissues. In contrast, in malignant tumor tissues CD276 expression levels are up-regulated. Moreover, higher expression levels of CD276 has been found to be correlated with poorer prognosis in cancer patients. Relevant studies have been conducted in a wide range of tumor types, including cancers of the bladder, breast, cervix, colorectal, esophageal, renal, hepatic, lung, ovarian, pancreatic, prostate, biliary, oral squamous cell carcinoma, intrauterine membranous cancer, squamous cell carcinoma, gastric cancer, glioma, melanoma, and adrenal malignancies (3-25) (shown as Table 1).

Genitourinary tumors provide a good example of elevated CD276 expression in tumor tissue. Xylinas et al. demonstrated that expression levels of PD-1 and CD276 were significantly higher in bladder urothelial carcinoma tissue when compared with adjacent normal tissues (3). In another study, Benzon et al. explored the association between CD276 expression and clinicopathologic variables. CD276 expression level was found to be positively correlated with Gleason score, tumor stage, castration resistance, and metastasis. They also performed a gene set enrichment analysis and results indicated CD276 was associated with androgen signaling as well as immune regulatory pathways (19). Another study conducted on a cohort of patients treated for clear cell renal cell carcinoma (ccRCC) reported that CD276 expression was detected in $17 \%$ of tumor cells and $95 \%$ of tumor vasculature. Expression of CD276 in ccRCC specimens is correlated with adverse clinicopathologic outcomes (tumor size, tumor stage, nuclear grading, coagulative tumor necrosis, and sarcomatoid differentiation). In multivariable analysis, the presence of CD276 in tumor cells or diffusely within tumor vasculature was significantly associated with an increased risk of death from ccRCC (21).

Abnormal CD276 expression has also been observed in rare tumors with a high degree of malignancy. Adrenocortical carcinoma (ACC) is characterized as a malignant adrenal tumor with a high recurrence rate and a short disease-free survival, and 91.7\% (44/48) of ACCs expressed cytoplasmic and membranous CD276 in neoplastic or tumor associated vascular cells. CD276 expression in tumor blood vessels was significantly positively correlated with local invasion (higher $\mathrm{T}$ stage) and with advanced ENSAT stage (European Network for the Study of Adrenal Tumors). Cytoplasmic CD276 expression is negatively correlated with both the overall survival and disease-free survival of ACC patients. Gene set enrichment analysis demonstrated that CD276 expression is closely related to immune regulation and malignant biological behavior of ACC (24).

TABLE 1 | The expression of CD276 in tumors and its relationship with pathological features.

\begin{tabular}{|c|c|c|c|}
\hline Tumor type & Expression & Clinical significance & Reference \\
\hline Bladder cancer & High & Not reported & (3) \\
\hline Endometrial cancer & High & Associated with poor prognosis and TNM stages & (4) \\
\hline Pancreatic cancer & High & $\begin{array}{l}\text { Associated with lymph node migration, tumor size, TNM stages, and cancer } \\
\text { recurrence }\end{array}$ & $(5,6)$ \\
\hline Cervical cancer & High & Associated with poor prognosis and tumor size & (7) \\
\hline Breast cancer & High & $\begin{array}{l}\text { Associated with poor prognosis, tumor size, TNM stages, lymph node migration, and } \\
\text { cancer recurrence }\end{array}$ & $(8,9)$ \\
\hline $\begin{array}{l}\text { Intrahepatic } \\
\text { cholangiocarcinoma }\end{array}$ & High & $\begin{array}{l}\text { Associated with poor prognosis, lymph node migration, tumor's progression, and } \\
\text { cancer recurrence }\end{array}$ & $(10)$ \\
\hline Colorectal cancer & High (membrane, cytoplasm, and nuclei) & $\begin{array}{l}\text { Associated with poor prognosis, TNM stages, lymph node migration, and cancer } \\
\text { recurrence }\end{array}$ & $(11)$ \\
\hline Ovarian cancer & $\begin{array}{l}\text { High (membrane, cytoplasm, and tumor } \\
\text { endothelium) }\end{array}$ & Associated with poor prognosis and TNM stages & $(12)$ \\
\hline Glioma & High & Associated with TNM stages & (13) \\
\hline Melanoma & High & Associated with poor prognosis and TNM stages & (14) \\
\hline Lung cancer & High & $\begin{array}{l}\text { Associated with poor prognosis, TNM stages, lymph node migration, and cancer } \\
\text { Recurrence }\end{array}$ & $(15,16)$ \\
\hline Liver cancer & High & $\begin{array}{l}\text { Associated with poor prognosis, lymph node migration, tumor's progression, and } \\
\text { TNM stages }\end{array}$ & $(17)$ \\
\hline Prostatic cancer & High & $\begin{array}{l}\text { Associated with poor prognosis, lymph node migration, tumor's progression, TNM } \\
\text { stages, and cancer recurrence }\end{array}$ & $(18,19)$ \\
\hline $\begin{array}{l}\text { Oral squamous cell } \\
\text { carcinoma }\end{array}$ & High & Associated with poor prognosis, tumor size, and TNM stages & $(20)$ \\
\hline Kidney cancer & High (tumor vessels and tumor cells) & Associated with poor prognosis, tumor size, and TNM stages & $(21)$ \\
\hline Pancreatic cancer & High & Positively correlated to the prognosis of patients & $(22)$ \\
\hline Gastric cancer & High & Positively correlated to the prognosis of patients & (24) \\
\hline $\begin{array}{l}\text { Adrenocortical } \\
\text { carcinoma }\end{array}$ & $\begin{array}{l}\text { High (membrane, cytoplasm, and tumor } \\
\text { vessels) }\end{array}$ & $\begin{array}{l}\text { Negatively correlated to the prognosis of patients Associated with tumor size and } \\
\text { TNM stages }\end{array}$ & $(25)$ \\
\hline
\end{tabular}


To give a more intuitive aspect of the expression level of CD276 in malignancies, we performed a pan-cancer analysis to evaluate CD276 expression level in multiple cancers on UALCAN database (http://ualcan.path.uab.edu/index.html). UALCAN is an online web-server for facilitating tumor subgroup gene expression and survival analyses based on TCGA RNA sequencing data and clinical data. CD276 expression level in cancer and corresponding normal tissues is shown Figure 1A. We also explored the correlation of CD276 expression with survival and found CD276 expression is significantly correlated with survival probability in a wide range of cancer types $(\mathrm{p}<0.05)$ (Figures $\mathbf{1 B}-\mathbf{K})$.

\section{Regulatory Mechanisms of CD276}

Although CD276 mRNA expression is detected in most human tissues, CD276 protein expression is not ubiquitous (26-28). The difference between CD276 mRNA and protein expression suggests that post-transcriptional regulation mechanisms may cause the discrepancy between transcription and translation (29, 30). Previously our understanding of the molecular mechanisms behind the regulation of CD276 expression was limited. Woong et al. (30) performed an in vivo study and found that in mouse dendritic cells CD276 expression could be enhanced by interferon- $\gamma$ (IFN- $\gamma$ ) and suppressed by interleukin 4 (IL-4). They concluded that CD276 was a negative regulator of T cell function preferentially affecting type- $1 \mathrm{~T}$ helper cell responses (30). $\mathrm{CD}^{+} \mathrm{CD} 25$ regulatory $\mathrm{T}$ cells (Tregs) have immunosuppressive effects and can inhibit the proliferation of effector $\mathrm{T}$ cells both in vivo and in vitro (31). Myeloid dendritic cells co-cultured with regulatory $\mathrm{T}$ cells are able to induce upregulation of $\mathrm{CD} 276$ expression, thereby affecting the maturation of dendritic cells (32). MicroRNAs (miRNAs) can also regulate the expression of CD276 through direct interaction with the $3^{\prime}$ UTR of CD276 mRNA and decreasing the level of protein translation $(33,34)$. miRNA-29 is highly expressed in normal tissues and poorly expressed in a variety of solid tumors, which is negatively correlated with CD276 expression. Luciferase reporter gene analysis shows a conserved miR-29-targeting site of CD276 in the 3 'UTR region $(35,36)$. In the study of CD276 in cutaneous melanoma, researchers found that MiR-29c expression could inversely regulate CD276 expression (14). However, the mechanism regulating the downstream signaling pathway by CD276 remains to be elucidated. Immunoglobulin-like transcript 4 (ILT4) is another molecule regulated by CD256. ILT4 belongs to the immunoglobulin-like super family that inhibit immune cell function (37). Research indicates that ILT4 mediated activation of $\mathrm{PI} 3 \mathrm{~K} / \mathrm{AKT} / \mathrm{mTOR}$ pathway increases CD276 expression in non-small cell lung cancer (NSCLC) (38). PDZ-binding kinase (PBK), whose expression is associated with immune infiltration in nasopharyngeal carcinoma (NPC), also plays an important role in CD276 expression regulation. In a recent study conducted by Wang et al., they demonstrated that the phosphorylation level of MSL1 was enhanced in PBKoverexpression cells and meanwhile the expression of CD276 was up-regulated. The up-regulation of CD276 following PBK-overexpression could be reversed by MSL1 depletion. Mechanistically, MSL1 serves as a mediator in the
PBK-MSL1-CD276 axis (39). Overall, there are many intertwined factors contributing to the regulation of CD276 expression, which result in the observed relatively low level expression in normal tissue and elevated expression in tumor tissue. Further investigations are required to develop our understanding of CD276 expression regulation.

\section{Potential Receptors of CD276}

The immune function of the majority of the B7 family is mediated by a variety of known receptors. For example, B7-1 or B7-2 bind to CD28 and CTLA-4, PD-L1 (B7-H1) or PD-L2 (B7-DC) bind to PD-1, and B7-H6 binds to NKp30 (40, 41). However, the receptor for CD276 has not been verified. According to Hashiguchi's findings, CD276 receptor may be expressed in a Trem-like transcript 2 (TLT-2 or TREML2) $(42,43)$. After introduction of TLT-2 into T cells, stimulating with CD276 resulted in increased production of interleukin-2 (IL-2) and IFN- $\gamma(37,42)$. CD276 was initially mooted as a ligand for TREM receptor family. However, subsequent studies showed contradictory results. A study by the Leitner group found no evidence for an interaction between CD276 and TREML2 in mouse or human cells (44). Furthermore, size exclusion chromatography (SEC) and surface plasmon resonance experiments demonstrated that murine CD276 and TLT-2 do not bind one another (45). Those contradictory results suggested that TLT-2 is unlikely to be a receptor for CD76, and an alternative receptor interacts with CD276 to mediate inhibitory immune responses.

In 2019, Husain et al. (46) implemented a new platform (conditioned medium AlphaScreen) to sensitively detect receptor-ligand interactions at high throughput. With the help of the technology, Husain identified interleukin receptor IL20RA as a receptor for the checkpoint inhibitor CD276. Further confirmatory studies about CD276 receptors are being conducted.

\section{CD276 AND IMMUNE CHECKPOINT SIGNALS}

CD276 was initially described as a co-stimulatory molecule for T cell activation (2). But subsequent research revealed that CD276 had a strong immunosuppressive effect, and could inhibit the proliferation of T cells (47). Furthermore, up-regulated CD276 promotes immune escaping of tumor cells, reducing the secretion of IFN- $\gamma$, tumor necrosis factor-alpha (TNF- $\alpha$ ), and other cytokines (48). Though a number of studies reported contradictory results and the receptor-mediated mechanism has not been clearly elucidated, mounting evidence demonstrates that CD276 has synergetic effects with other immune checkpoints.

\section{CD276 and PD-1}

Programmed Cell Death-1 (PD-1) is an immunosuppressive receptor, first reported in 1992 (49). PD-1, also known as B7H1 and CD279, is encoded by PDCD1 gene on chromosome 2. PD-1 is a member of CD28 super family and is located on $\mathrm{T}$ cell and B cell surfaces. CD28 family members 
Lir et al.

Progress of CD276 in Cancers

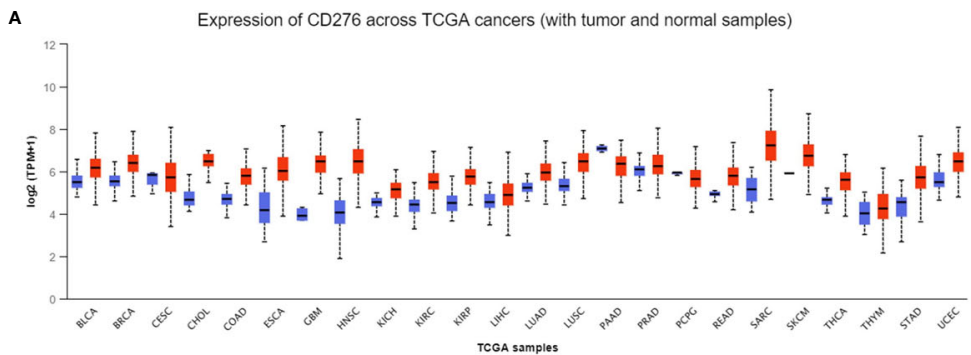

B

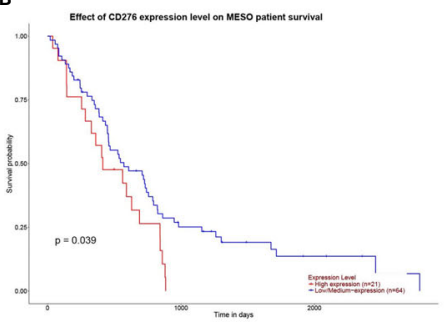

D
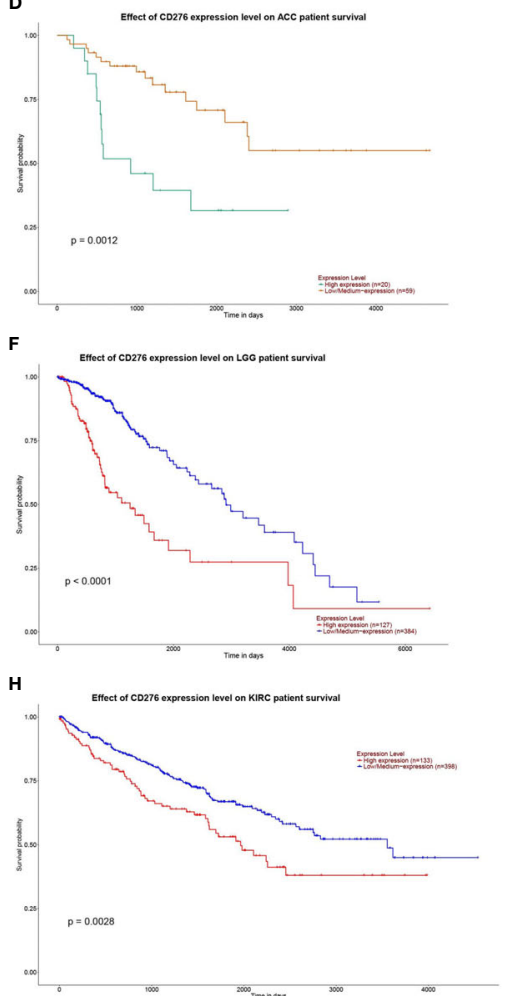

$\mathbf{J}$



c
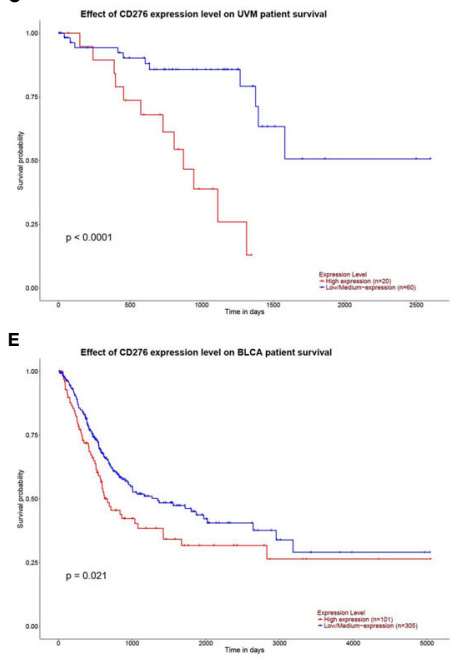

G
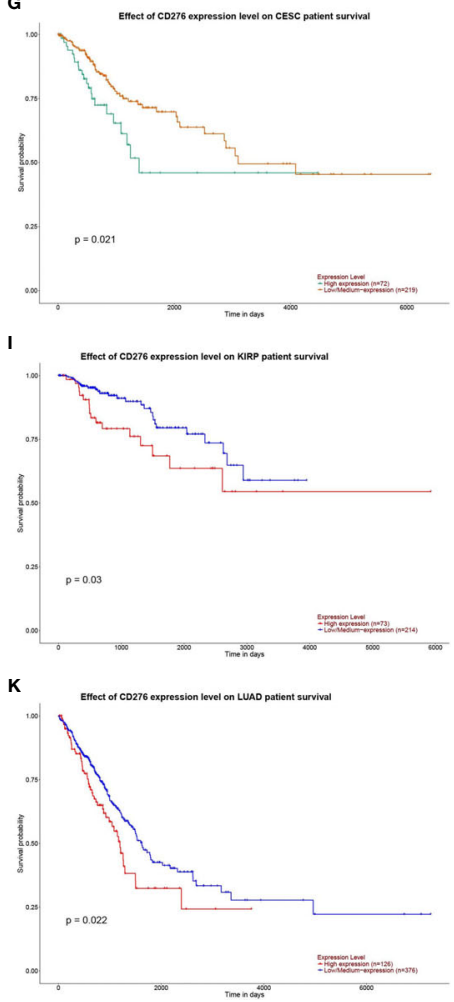

FIGURE 1 | (A): CD276 expression level in cancer and corresponding normal tissues. (B-K): The correlation of CD276 expression with survival in cancer patients $(p<0.05)$.

Frontiers in Oncology | www.frontiersin.org

4

March 2021 | Volume 11 | Article 654684 
play an important role in the regulation of T cell activity through interaction with ligands and transmission of inhibitory signals (50). PD-1 can interact with PD-L1 (B7-H1) and PD-L2 (B7$\mathrm{DC})$, thereby down-regulating the activity of effector $\mathrm{T}$ cells in normal tissues and tumors $(51,52)$.

$\mathrm{PD}-1$ is one of the checkpoint receptors, which is tightly associated with immune escaping of tumor cells. Such a characteristic renders PD-1/PD-L1/PD-L2 as a significant target for immunotherapy, which has been applied to cancer therapy and promising results were reported. Even so, PD-1/PDL1/PD-L2 molecules are still only a small part of immunological checkpoints with therapeutic values. There are more potential immune checkpoints which may be used as therapeutic targets. PD-1 and CD276 are both members of B7/CD28 family and had similar effects on the tumor growth micro-environment. The binding of CD276/PD-1 and corresponding receptors inhibits the proliferation of $\mathrm{T}$ cells and the secretion of IFN- $\gamma$, TNF- $\alpha$, and other cytokines, exerting synergistic effect with PD-1/PDL1/PD-L2 (47, 48). Combination therapy with anti-CD276 antibody and anti-PD1/PD-L1 antibody for tumor treatment is another hot topic of research. In a recent study, it was reported that Photodynamic therapy (PDT) combined with PD1/PD-L1 and CD276 antibody respectively successfully improves the tumor suppressive blocking effect of PD-1/PD-L1 (53).

\section{Relationship Between CD276 and CTLA4}

Another promising molecule for immune regulation is CTLA4 (CD152). CTLA4 is an intracellular glycoprotein that acts as a functional inhibitor of T cell responses (54). Mechanically, on the surface of dendritic antigen presenting cells, CD80/CD86 binds to CD28 on adjacent $\mathrm{T}$ cells, thereby activating $\mathrm{T}$ cells and producing cytokines. This interactive process is involved in multiple acute or chronic inflammatory diseases, such as atherosclerosis (55), autoimmune diseases (56), and malignant tumors (57). CTLA4 is a homologue of CD28, which has stronger binding affinity than CD28. CTLA4 can attenuate T cell activation once combined with antigen-presenting cells (APC) (58). Moreover, CTLA4 can reduce the contractive time between $\mathrm{T}$ cells and antigen-presenting cells. Therefore, an immunosuppressive microenvironment such as that mediated by CTLA4 can reduce the CD28-CD80/CD86 interaction time, and thus reduce $\mathrm{T}$ cell activation (59).

Based on the information above, CD276 inhibits T cell activation and has synergistic effects with CTLA4. Leitner et al. believe that CD276 exerts its inhibitory effect on T cells by inhibiting IL-2 secretion. Exogenous supplementation with IL-2 can reverse this effect, suggesting that CD276 acts as an upstream regulatory molecule for IL-2 (44). CTLA4 is expressed in normal tissues, normal immune cells, and tumor cells simultaneously. Compared to CTLA4 (60), CD276 is only expressed in tumors and tumor-related cells. Therefore, CD276 has higher specificity for tumor tissues.

\section{ROLE OF CD276 IN TUMORIGENESIS}

CD276 can directly participate in the regulation of immunity and various malignant behaviors of tumor cells.

\section{CD276 in Tumor Cells}

CD276 is involved in the regulation of proliferation, apoptosis, invasion, cell cycle, cell differentiation, cell autophagy, and epithelial-mesenchymal transition. To examine the role of CD276 in metabolic reprogramming of cancer cells, Lim et al. (61) conducted a study and found that tumor cells expressing CD276 significantly increase glucose uptake and lactate production both in vivo and in vitro. Thus, even in aerobic environments, cancer cells still produce energy by metabolizing glucose into lactic acid (anaerobic glycolysis), which is called Warburg effect and this is promoted by CD276. CD276 also increases the expression level of HIF-1 $\alpha$, as well as the expression of its downstream signaling molecules and the key enzymes in the glycolysis pathway (LDHA and PDK1). Furthermore, it is reported that $\mathrm{CD} 276$ promotes the stability of active oxygendependent HIF-1 $\alpha$ by inhibiting the stress-activated transcriptional factor (Nrf2) and its targets (antioxidants SOD1, SOD2, and PRX3). Finally, a metabolic imaging experiment further confirmed that CD276 enhances the glucose uptake by tumor cells, thereby promoting tumor growth in a mouse breast cancer xenograft model. These findings provided a new perspective of immunomodulatory B7 family on tumor progression.

CD276 participates in tumor metastasis. The ability of melanoma cells to invade the gel decreases by silencing CD276 using shRNA. In vivo, silencing CD276 with shRNA prolongs the asymptomatic survival period. CD276 shRNA inhibits the expression of metastasis-related proteins in melanoma cells, such as matrix metalloproteinase MMP-2, tissue inhibitors of metalloproteinase (TIMP1 and TIMP2), signal transduction and transcription activator (STAT3), and IL-8 (62). Kang et al. suggest that CD276 determines the malignancy and invasive ability of liver cancers through the JAK3/STAT3/SLUG signaling pathway involving MMP2. The expression of epithelial-derived marker E-cadherin is down-regulated in epithelial-mesenchymal transition (EMT). The expression of E-cadherin in CD276knockout liver cancer cells is increased significantly suggesting that CD276 plays a key role in EMT, which is enables the metastasis of liver cancer cells (63).

There is a correlation between the expression of MYC and CD276. Knocking out CD276 regulates the differentiation of malignant gliomas by increasing/decreasing expression of MYC, which causes silencing of SMAD6 (TGF- $\beta$ pathway inhibitor) and enhanced SMAD4 expression (64). In vivo, CD276 knockout decreases the expression of MYC and inhibits tumor growth (64). In another study, down-regulation of CD276 renders the resistance of gastric cancer cells to radiotherapy by regulating apoptosis, cell cycle progression and DNA double-strand breaks. Furthermore, CD276 regulates the baseline level of autophagy. Adding rapamycin to CD276-up-regulated cells increases the baseline level of autophagy and enhances the sensitivity of radiotherapy (65).

\section{The Role of CD276 in Tumor Vessels}

Nutrition is essential for tumor cell proliferation. New blood vessels are required in tumors to ensure substrate provision to cells distant from the normal vasculature. Normal tissue blood 
supply cannot guarantee the stable support of tumors. Therefore, angiogenesis markers are considered to be another characteristic of tumors (66). Angiogenesis inhibitors are used to suppress tumor progression. Researchers have demonstrated that tumors generate numerous new blood vessels in glioblastoma models (67). In addition, by selectively targeting endothelial cells produced by tumor stem cells, the researchers found that the number of tumor cells was significantly reduced and degraded. This phenomenon suggests that anti-angiogenesis is a potential target for anti-tumor therapy (68).

Recently, a number of studies have shown that CD276 is expressed in tumor vascular endothelial cells, suggesting that CD276 is related to tumor angiogenesis. It is reported that CD276 is expressed in the vascular system of renal tumors (21). Positive expression of CD276 is also observed in gastric adenocarcinoma blood vessels (24). CD276 is widely observed in vascular endothelial cells of colon, lung, and breast cancers (25). Importantly, CD276 is present only in the pathological blood vessels and is absent in physiological blood vessels. In addition to its expression in the tumor vascular system, CD276 also affects the tumor microenvironment through microvessels in a soluble form (sB7$\mathrm{H} 3$ ). ELISA analysis showed that the expression level of sB7-H3 is elevated in the supernatant of pancreatic cancer cell culture, indicating that pancreatic cancer cells release sB7-H3 into the extracellular medium. Next, the researchers exposed pancreatic cancer cells to sB7-H3, finding that sB7-H3 significantly increased the migration and invasion of cancer cells (69). Furthermore, Lai et al. (70) studied the role of CD276 in angiogenesis using human umbilical vein endothelial cells (HUVECs). In vitro and in vivo experiments demonstrated that CD276 promotes the secretion of vascular endothelial growth factor (VEGF), which further promotes the formation of blood vessels. CD276 could also activate NF- $\mathrm{KB}$ signaling through a TLR-4-dependent mechanism, and this could cause subsequent up-regulation of VEGF and IL-8. Elevated expression levels of VEGF and IL-8 further promote tumor invasion and angiogenesis (71). In general, CD276s role in tumor angiogenesis should be a topic of concern and is worthy of further research. It is expected to be a new target for anti-tumor angiogenesis.

\section{CD276 AND IMMUNOTHERAPY}

High hopes have been invested in immunotherapy for treatment of tumors in recent years. Immune checkpoints inhibitors (ICIs) targeting PD-1/PD-L1, CTLA-4 have been brought into clinical application and great efficacy was shown for numerous malignant tumors. As CD276 expression level was observed to be correlated to tumor growth, invasion, and metastasis, CD276 could be a potential target for immunotherapy. In previous studies, CD276 targeted therapies including monoclonal antibodies blocking CD276, CD276 specific antibody-dependent cell-mediated cytotoxicity (ADCC), antibody drug conjugates (ADC); bispecific antibodies against CD276/CD3, CD276-specific small-molecule inhibitor, and chimeric antigen receptor (CAR) T-cell therapy have been discussed $(1,34,72,73)$. In this review, we investigated the mechanisms underlying therapies targeting the immune checkpoint CD276 (Figure 2).

\section{CD276 and Gene Therapy}

Drug vectors targeting the expression of CD276 may be an ideal therapeutic approach. Lupu et al. (74) injected the adenovirus vector expressing $\mathrm{mB} 7-\mathrm{H} 3 \mathrm{CD} 276$ into colon tumors resulting in reduction of the primary tumor volume and secondary metastases. Yang et al. (75) demonstrated that transfecting CD276 plasmids into oral squamous cell carcinomas simultaneously enhances the proliferation of tumor cells, production of interferon, and the activity of cytotoxic $\mathrm{T}$ cells. Vasostatin is an effective inhibitor of angiogenesis. The combination of vasodilator and CD276-pcDNA3.1 inhibits tumor angiogenesis and promotes leukocyte infiltration in hepatocellular carcinoma (HCC). Notably, the combination is more effective than a single drug (76). Though promising CD276 is as a potential gene target in anti-tumor therapy, the negative effects of CD276 regulation should be considered before the clinical transformation. In addition, the best mechanism of drug delivery remains to be determined.

\section{Inhibitory Treatment With CD276 Antibody}

To date, anti-CD276 antibodies have been developed, tested in animals, and are in clinical trials as immunotherapy agents. $\mathrm{Xu}$ et al. (36) confirmed that the antigen of monoclonal antibody $8 \mathrm{H} 9$ is $4 \mathrm{Ig}-\mathrm{B} 7-\mathrm{H} 3$ (CD276). After CD276 is blocked with $8 \mathrm{H} 9$, anti-tumor effects are witnessed. When $8 \mathrm{H} 9$ is further combined with miR-29, the anti-tumor immune response is further enhanced. MJ18, another anti-CD276 monoclonal antibody, significantly promotes the infiltration of CD8+ T cells and inhibits the growth of pancreatic tumors (6). Loo et al. (77) synthesized MGA271, an Fc-engineered monoclonal antibody. MGA271 inhibition of CD276 in RCC and bladder cancer in tumor xenograft models resulted in inhibition of tumor growth. Currently, 8H9 and MGA271 are undergoing clinical trials (shown as Table 2).

MAb 376.96 is another anti-CD276 monoclonal antibody that recognizes the expression of CD276 epitopes on differentiated ovarian cancer cells and chemotherapy-resistant cancer cells (78). In vitro studies showed that mAb 376.96 combined with the tyrosine kinase inhibitor sunitinib significantly inhibits tumor growth. Ma et al. (48) constructed an anti-CD3 and anti-CD276 antibody (B7-H3Bi-Ab) targeting both $\mathrm{T}$ cell receptors and tumor-related antigens. $\mathrm{B} 7-\mathrm{H} 3 \mathrm{Bi}-\mathrm{Ab}$ bound to activated T-cells (ATC) significantly enhanced production of IFN- $\gamma$, TNF- $\alpha$, and IL-2. Increased cytotoxicity mediated by $\mathrm{B} 7-\mathrm{H} 3 \mathrm{Bi}-\mathrm{Ab}$ is shown in different human tumor cell lines and in primary cells isolated from patients with breast and lung cancer. In vivo, experiments showed that, compared with ordinary activated $\mathrm{T}$ cells, B7-H3Bi-Ab assembled ATCs significantly inhibited tumor growth and prolonged survival (48). Seaman et al. reported that CD276-targeted antibody conjugated with drugs acting on tumor cells and tumor blood vessels simultaneously. In addition, m276-PBD dimer has shown extensive tumor killing effects and antimetastasis activity in vivo (25). In a recent study conducted by 


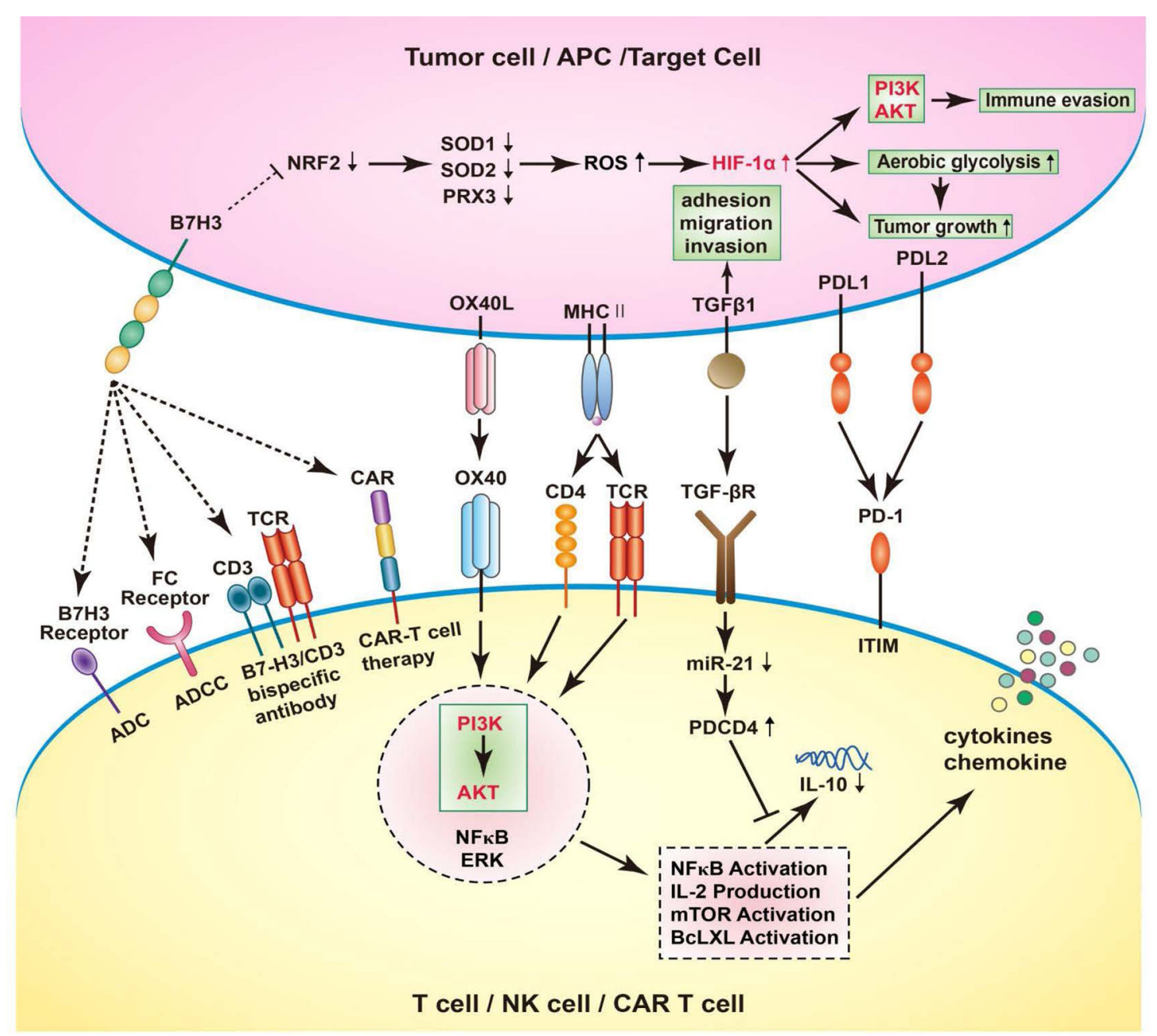

FIGURE 2 | B7-H3 increases ROS and HIF-1 $\alpha$ through B7-H3-induced Nrf2 suppression, SOD1, SOD2, and PRX3 reduction, thus promote aerobic glycolysis in cancer cells, leading to tumor growth. Human cancer immunotherapy strategies targeting B7-H3 including blockade of B7-H3 with blocking monoclonal antibodies (mAbs); B7H3 specific antibody-dependent cell-mediated cytotoxicity (ADCC); CD3/B7-H3 bispecific antibodies; Small molecule inhibitors; Engineered chimeric antigen receptor (CAR) T cells; combination with other therapies. In T cells, OX40 engagement by OX40L forms a signaling complex with a number of established pro-inflammatory mediators, including AKT, PI3K, NFKB, ERK. Through the PI3K/AKT signaling pathway, the downstream signatures were activated, such as NFkB activation, IL-2 production, mTOR activation, BcLXL activation. As a result, NFkB activation stimulates the expression of chemokines and cytokines, including IL-10. Interestingly, the activation of TGFB1 receptor could simultaneously suppress the maturation of miR-21 and enhance PDCD4 levels. Consequently, the translation of the anti-inflammatory cytokine IL-10 is inhibited. In RCC cells, the TGFBI could participate in the adhesion, migration, and invasion, depending on the inactivation of VHL.

Nathan et al. (79) a new CD276 targeting ADC m276-SL-PBD was introduced and its activity was explored in both pediatric solid malignancy patient-derived xenograft (PDX) and cell linederived xenograft (CDX) models. Significant anti-tumor activity of m276-SL-PBD was observed in PDX models of solid tumors including Ewing sarcoma, rhabdomyosarcoma, Wilms tumors, osteosarcoma, and neuroblastoma. Another anti-CD276 ADC, MGC018 also shows antitumor activity across a range of human tumor xenografts (80).

Results of these studies suggest that CD276-targeted inhibitors may be an exciting immunotherapy strategy in the future.

\section{CD276-Chimeric Antigen Receptor (CAR) T Cell Therapy}

Chimeric antigen receptor (CARs) $\mathrm{T}$ cell therapy is popular in immunotherapy. The objective response rate of CAR-T cell therapy in patients with hematologic malignancy is high. In solid tumors, due to the lack of tumor-specific antigens and the immunosuppressive effect of tumor microenvironment, there are some obstacles for CAR T cells to overcome. The gene module programming technology can improve the efficacy and specificity of CAR T, which is expected to target solid tumors for CAR T cells.

In 2019, Du et al. (81) constructed CAR-T cells targeting CD276. They found that CD276 CAR T cells control tumor growth in vitro 
TABLE 2 | CD276 related clinical trials.

Relapsed and refractory neuroblastoma (NB)

Advanced Solid Tumors

Melanoma, Non-small Cell Lung Cancer

and in vivo. CD276 CAR-T showed stronger anti-tumor activity when targeted at tumor cells expressing PD-L1. Du et al. believed that CAR T cells significantly inhibit tumor growth without obvious toxicity or side effects. These evidence all support the prospect of CD276 CAR-T cell therapy being promising in patients. CAR-T cell therapy has shown great success in the treatment of recurrent childhood acute lymphocytic leukemia. However, due to the lack of reliable, targeted, and differentially expressed molecular cell surface markers in solid tumors, CAR-T cell technology has not yet been applied. Majzner et al. (82) developed a new CD276 CAR-T cell which binds to a monoclonal antibody. The antibody preferentially binds to tumor tissues and safety has been tested in clinical trials. By detecting CD276 CAR-T cells in a variety of childhood cancer models, Majzner et al. found that CD276 CAR-T cells exhibit antitumor activity in vivo, leading to regression of solid tumors in xenograft models including osteosarcoma, medulloblastoma, and Ewing's sarcoma. They also demonstrated that the efficacy of CD276 CAR-T cells depended on the density of target antigens on the tumor surface, while the activity of cells expressing low levels of antigens is significantly reduced. In a recent study, Johanna et al. (83) found CD276 CAR T cells were highly active against atypical teratoid/rhabdoid tumors, for which most current therapies have been proven to be ineffective. In summary, increased expression level of CD276 in tumor tissues compared to normal tissues provides the potential for CD276 CAR-T cells as a treatment for tumors.

\section{CONCLUSION}

The functionality of CD276 is complicated. Although great progress has been made in the past two decades, further research is still needed. The regulation of CD276 expression is closely related to tumor growth, invasion, metastasis, and immune escape. Due to increased expression levels of CD276 in multiple tumors and low expression in a variety of normal tissues, numerous studies used CD276 as the target for tumor gene therapy and monoclonal antibody therapy. Overall, CD276 does have great potential as an immunotherapeutic target, but its clinical value must be confirmed by further studies and clinical trials.

\section{AUTHOR CONTRIBUTIONS}

Conception and design: XW and YL. Acquisition of data: SL and JL. Analysis and interpretation of data: ZL, CZha, YW, and CZho. Drafting of the manuscript: SL and JL. Critical revision of the manuscript for important intellectual content: XW and YL. Administrative, technical, or material support: AW, FZ, KW, and FXZ. Supervision: XW and YL. All authors contributed to the article and approved the submitted version.

\section{FUNDING}

This paper was supported by the following grants: China Scholarship Council 202006240301 to SL.

\section{SUPPLEMENTARY MATERIAL}

The Supplementary Material for this article can be found online at: https://www.frontiersin.org/articles/10.3389/fonc.2021.654684/ full\#supplementary-material 


\section{REFERENCES}

1. Flem-Karlsen K, Fodstad $\varnothing$, Tan M, Nunes-Xavier CE. B7-H3 in cancerbeyond immune regulation. Trends Cancer (2018) 4:401-4. doi: 10.1016/ j.trecan.2018.03.010

2. Chapoval AI, Ni J, Lau JS, Wilcox RA, Flies DB, Liu D, et al. B7-H3: a costimulatory molecule for $\mathrm{T}$ cell activation and IFN- $\gamma$ production. Nat Immunol (2001) 2:269-74. doi: 10.1038/85339

3. Xylinas E, Robinson B, Kluth L, Volkmer BG, Hautmann R, Küfer R, et al. Association of T-cell co-regulatory protein expression with clinical outcomes following radical cystectomy for urothelial carcinoma of the bladder. Eur J Surg Oncol (2014) 40:121-7. doi: 10.1016/j.ejso.2013.08.023

4. Brunner A, Hinterholzer S, Riss P, Heinze G, Brustmann H. Immunoexpression of B7-H3 in endometrial cancer: relation to tumor Tcell infiltration and prognosis. Gynecol Oncol (2012) 124:105-11. doi: 10.1016/j.ygyno.2011.09.012

5. Zhao X, Li D-C, Zhu X-G, Gan W-J, Li Z, Xiong F, et al. B7-H3 overexpression in pancreatic cancer promotes tumor progression. Int $\mathrm{J} \mathrm{Mol}$ Med (2013) 31:283-91. doi: 10.3892/ijmm.2012.1212

6. Yamato I, Sho M, Nomi T, Akahori T, Shimada K, Hotta K, et al. Clinical importance of B7-H3 expression in human pancreatic cancer. $\mathrm{Br} J$ Cancer (2009) 101:1709-16. doi: 10.1038/sj.bjc.6605375

7. Huang C, Zhou L, Chang X, Pang X, Zhang H, Zhang S. B7-H3, B7-H4, Foxp3 and IL-2 expression in cervical cancer: Associations with patient outcome and clinical significance. Oncol Rep (2016) 35:2183-90. doi: 10.3892/or.2016.4607

8. Maeda N, Yoshimura K, Yamamoto S, Kuramasu A, Inoue M, Suzuki N, et al. Expression of B7-H3, a potential factor of tumor immune evasion in combination with the number of regulatory $\mathrm{T}$ cells, affects against recurrence-free survival in breast cancer patients. Ann Surg Oncol (2014) 21:546-54. doi: 10.1245/s10434-014-3564-2

9. Arigami T, Narita N, Mizuno R, Nguyen L, Ye X, Chung A, et al. B7-H3 ligand expression by primary breast cancer and associated with regional nodal metastasis. Ann Surg Oncol (2010) 252:1044-51. doi: 10.1097/ SLA.0b013e3181f1939d

10. Cheng R, Chen Y, Zhou H, Wang B, Du Q, Chen Y. B7-H3 expression and its correlation with clinicopathologic features, angiogenesis, and prognosis in intrahepatic cholangiocarcinoma. APMIS (2018) 126:396-402. doi: 10.1111/ apm. 12837

11. Ingebrigtsen VA, Boye K, Tekle C, Nesland JM, Flatmark K, Fodstad O, et al. B7H3 expression in colorectal cancer: nuclear localization strongly predicts poor outcome in colon cancer. Int J Cancer (2012) 131:2528-36. doi: 10.1002/ijc.27566

12. Zang X, Sullivan PS, Soslow RA, Waitz R, Reuter VE, Wilton A, et al. Tumor associated endothelial expression of B7-H3 predicts survival in ovarian carcinomas. Mod Pathol (2010) 23:1104-12. doi: 10.1038/modpathol.2010.95

13. Zhou Z, Luther N, Ibrahim GM, Hawkins C, Vibhakar R, Handler MH, et al. $\mathrm{B} 7-\mathrm{H} 3$, a potential therapeutic target, is expressed in diffuse intrinsic pontine glioma. J Neurooncol (2013) 111:257-64. doi: 10.1007/s11060-012-1021-2

14. Wang J, Chong KK, Nakamura Y, Nguyen L, Huang SK, Kuo C, et al. B7-H3 associated with tumor progression and epigenetic regulatory activity in cutaneous melanoma. J Invest Dermatol (2013) 133:2050-8. doi: 10.1038/ jid.2013.114

15. Inamura K, Yokouchi Y, Kobayashi M, Sakakibara R, Ninomiya H, Subat S, et al. Tumor B7-H3 (CD276) expression and smoking history in relation to lung adenocarcinoma prognosis. Lung Cancer (2016) 243:21-8. doi: 10.1016/ j.lungcan.2016.09.016

16. Sun Y, Wang Y, Zhao J, Gu M, Giscombe R, Lefvert AK, et al. B7-H3 and B7H4 expression in non-small-cell lung cancer. Lung Cancer (2006) 53:143-51. doi: 10.1016/j.lungcan.2006.05.012

17. Sun T-W, Gao Q, Qiu S-J, Zhou J, Wang XY, Yi Y, et al. B7-H3 is expressed in human hepatocellular carcinoma and is associated with tumor aggressiveness and postoperative recurrence. Cancer Immunol Immunother (2012) 61:217182. doi: $10.1007 / \mathrm{s} 00262-012-1278-5$

18. Zang X, Thompson RH, Al-Ahmadie HA, Serio AM, Reuter VE, Eastham JA, et al. B7-H3 and B7x are highly expressed in human prostate cancer and associated with disease spread and poor outcome. Proc Natl Acad Sci USA (2007) 104:19458-63. doi: 10.1073/pnas.0709802104

19. Benzon B, Zhao S, Haffner M, Takhar M, Erho N, Yousefi K, et al. Correlation of B7-H3 with androgen receptor, immune pathways and poor outcome in prostate cancer: an expression-based analysis. Prostate Cancer Prostatic Dis (2017) 20:28-35. doi: 10.1038/pcan.2016.49

20. Chen J-T, Chen C-H, Ku K-L, Hsiao M, Chiang CP, Hsu TL, et al. Glycoprotein B7-H3 overexpression and aberrant glycosylation in oral cancer and immune response. Proc Natl Acad Sci USA (2015) 112:1305762. doi: $10.1073 /$ pnas. 1516991112

21. Crispen PL, Sheinin Y, Roth TJ, Lohse CM, Kuntz SM, Frigola X, et al. Tumor cell and tumor vasculature expression of $\mathrm{B} 7-\mathrm{H} 3$ predict survival in clear cell renal cell carcinoma. Clin Cancer Res (2008) 14:5150-7. doi: 10.1158/10780432.CCR-08-0536

22. Loos M, Hedderich DM, Ottenhausen M, Giese NA, Laschinger M, Esposito I, et al. Expression of the costimulatory molecule B7-H3 is associated with prolonged survival in human pancreatic cancer. BMC Cancer (2009) 9:1-10. doi: 10.1186/1471-2407-9-463

23. Dai W, Shen G, Qiu J, Zhao X, Gao Q. Aberrant expression of B7-H3 in gastric adenocarcinoma promotes cancer cell metastasis. Oncol Rep (2014) 32:208692. doi: 10.3892/or.2014.3405

24. Liang J, Liu Z, Pei T, Xiao Y, Zhou L, Tang Y, et al. Clinicopathological and Prognostic Characteristics of CD276 (B7-H3) Expression in Adrenocortical Carcinoma. Dis Markers (2020) 2020:5354825. doi: 10.1155/2020/5354825

25. Seaman S, Zhu Z, Saha S, Zhang XM, Yang MY, Hilton MB, et al. Eradication of tumors through simultaneous ablation ofCD276/B7-H3-positive tumor cells and tumor vasculature. Cancer Cell(2017) 31:501-15.e508. doi: 10.1016/ j.ccell.2017.03.005

26. Flem-Karlsen K, Fodstad $\varnothing$, Nunes-Xavier CE, et al. B7-H3 Immune Checkpoint Protein in Human Cancer.Curr Med Chem (2020)27(24):406286. doi: 10.2174/0929867326666190517115515

27. Greenwald RJ, Freeman GJ, Sharpe AH. The B7 family revisited. Annu Rev Immunol (2005) 23:515-48. doi: 10.1146/annurev.immunol.23.021704.115611

28. Yi KH, Chen LJIR. Fine tuning the immune response through B7-H3 and B7H4. Immunol Rev (2009) 229:145-51. doi: 10.1111/j.1600-065X.2009.00768.x

29. Hofmeyer KA, Ray A, Zang X. The contrasting role of B7-H3. Proc Natl Acad Sci USA (2008) 105:10277-8. doi: 10.1073/pnas.0805458105

30. Suh W-K, Gajewska BU, Okada H, Gronski MA, Bertram EM, Dawicki W, et al. The B7 family member B7-H3 preferentially down-regulates T helper type 1-mediated immune responses. Nat Immunol (2003) 4:899-906. doi: $10.1038 /$ ni967

31. Shevach EM, McHugh RS, Piccirillo CA, Thornton AM. Control of T-cell activation by CD4+ CD25+ suppressor T cells. Immunol Rev (2001) 182:5867. doi: 10.1034/j.1600-065X.2001.1820104.x

32. Mahnke K, Ring S, Johnson TS, Schallenberg S, Schönfeld K, Storn V, et al. Induction of immunosuppressive functions of dendritic cells in vivo by CD4+ $\mathrm{CD} 25+$ regulatory T cells: role of $\mathrm{B} 7-\mathrm{H} 3$ expression and antigen presentation. Eur J Immunol (2007) 37:2117-26. doi: 10.1002/eji.200636841

33. Bartel DP. MicroRNAs: genomics, biogenesis, mechanism, and function. Cell (2004) 116:281-97. doi: 10.1016/S0092-8674(04)00045-5

34. Dong P, Xiong Y, Yue J, Hanley SJB, Watari H. B7H3 as a promoter of metastasis and promising therapeutic target. Front Oncol (2018) 8:264. doi: 10.3389/fonc. 2018.00264

35. Bing G HC, Zhengpeng S. MiR-29a inhibited costimulatory molecule B7-H3 expression and theinvasion of glioma growth. Chin J Cancer Biother (2015) 22:28-33. doi: 10.3872/j.issn.1007-385X.2015.1.005

36. Xu H, Cheung IY, Guo H-F, Cheung N-KV. MicroRNA miR-29 modulates expression of immunoinhibitory molecule B7-H3: potential implications for immune based therapy of human solid tumors. Cancer Res (2009) 69:6275-81. doi: 10.1158/0008-5472.CAN-08-4517

37. Colonna M, Nakajima H, Cella M. A family of inhibitory and activating Ig-like receptors thatmodulate function of lymphoid and myeloid cells. Semin Immunol.(2000) 12(2):121-7. doi:10.1006/smim.2000.0214

38. Zhang P, Yu S, Li H, Liu C, Li J, Lin W, et al. ILT4 drives B7-H3 expression via $\mathrm{PI} 3 \mathrm{~K} / \mathrm{AKT} / \mathrm{mTOR}$ signalling and ILT4/B7-H3 co-expression correlates with poor prognosis in non-small cell lung cancer. FEBS Lett (2015) 589:2248-56. doi: 10.1016/j.febslet.2015.06.037

39. Wang M-Y, Qi B, Wang F, Li MY, Yin WJ. PBK phosphorylates MSL1 to elicit epigenetic modulation of CD276 in nasopharyngeal carcinoma. Oncogenesis (2021) 10:9. doi: 10.1038/s41389-020-00293-9

40. Linsley PS, Greene JL, Brady W, Bajorath J, Ledbetter JA, Peach R. Human B71 (CD80) and B7-2 (CD86) bind with similar avidities but distinct kinetics to 
CD28 and CTLA-4 receptors. Immunity (1994) 1:793-801. doi: 10.1016/ S1074-7613(94)80021-9

41. Brandt CS, Baratin M, Yi EC, Kennedy J, Gao Z, Fox B, et al. The B7 family member B7-H6 is a tumor cell ligand for the activating natural killer cell receptor NKp30 in humans. J Exp Med (2009) 206:1495-503. doi: 10.1084/ jem. 20090681

42. Hashiguchi M, Kobori H, Ritprajak P, Kamimura Y, Kozono H, Azuma M, et al. Triggering receptor expressed on myeloid cell-like transcript 2 (TLT-2) is a counter-receptor for B7-H3 and enhances T cell responses. Proc Natl Acad Sci USA (2008) 105:10495-500. doi: 10.1073/pnas.0802423105

43. Hashiguchi M, Inamochi Y, Nagai S, Otsuki N, Piao J, Kobori H, et al. Human B7-H3 binds to Triggering receptor expressed on myeloid cells-like transcript 2 (TLT-2) and enhances T cell responses. Open J Immunol (2012) 2:9. doi: 10.4236/oji.2012.21002

44. Leitner J, Klauser C, Pickl WF, Stöckl J, Majdic O, Bardet AF, et al. B7-H3 is a potent inhibitor of human T-cell activation: no evidence for B7-H3 and TREML2 interaction. Eur J Immunol (2009) 39:1754-64. doi: 10.1002/ eji.200839028

45. Vigdorovich V, Ramagopal UA, Lázár-Molnár E, Sylvestre E, Lee JS, Hofmeyer KA, et al. Structure and T cell inhibition properties of B7 family member, B7-H3. Structure (2013) 21:707-17. doi: 10.1016/j.str.2013.03.003

46. Husain B, Ramani SR, Chiang E, Lehoux I, Paduchuri S, Arena TA, et al. A platform for extracellular interactome discovery identifies novel functional binding partners for the immune receptors B7-H3/CD276 and PVR/CD155. Mol Cell Proteomics (2019) 18:2310-23. doi: 10.1074/mcp.TIR119.001433

47. Loos M, Hedderich DM, Friess H, Kleeff J. B7-h3 and its role in antitumor immunity.Clin Dev Immunol (2010) 2010:683875. doi: 10.1155/2010/683875

48. Ma J, Ma P, Zhao C, Xue X, Han H, Liu C, et al. B7-H3 as a promising target for cytotoxicity T cell in human cancer therapy. Oncotarget (2016) 7:29480. doi: 10.18632/oncotarget.8784

49. Ishida Y, Agata Y, Shibahara K, Honjo T. Induced expression of PD-1, a novel member of the immunoglobulin gene superfamily, upon programmed cell death. EMBO J (1992) 11:3887-95. doi: 10.1002/j.1460-2075.1992.tb05481.x

50. Agata $Y$, Kawasaki A, Nishimura H, Ishida $Y$, Tsubata T, Yagita H, et al. Expression of the PD-1 antigen on the surface of stimulated mouse T and B lymphocytes. Int Immunol (1996) 8:765-72. doi: 10.1093/intimm/8.5.765

51. Butte MJ, Keir ME, Phamduy TB, Sharpe AH, Freeman GJ. Programmed death-1 ligand 1 interacts specifically with the B7-1 costimulatory molecule to inhibit T cell responses. Immunity (2007) 27:111-22. doi: 10.1016/ j.immuni.2007.05.016

52. Mischinger J, Comperat E, Schwentner C, Stenzl A, Gakis G. Inflammation and cancer: what can we therapeutically expect from checkpoint inhibitors? Curr Urol Rep (2015) 16:1-6. doi: 10.1007/s11934-015-0532-8

53. Bao R, Wang Y, Lai J, Zhu H, Zhao Y, Li S, et al. Enhancing anti-PD-1/PD-L1 immune checkpoint inhibitory cancer therapy by CD276-targeted photodynamic ablation of tumor cells and tumor vasculature. Mol Pharm (2018) 16:339-48. doi: 10.1021/acs.molpharmaceut.8b00997

54. Linsley PS, Bradshaw J, Greene J, Peach R, Bennett KL, Mittler RS. Intracellular trafficking of CTLA-4 and focal localization towards sites of TCR engagement. Immunity (1996) 4:535-43. doi: 10.1016/S1074-7613(00) 80480-X

55. Ewing M, Karper J, Abdul S, de Jong RC, Peters HA, de Vries MR, et al. T-cell co-stimulation by CD28-CD80/86 and its negative regulator CTLA-4 strongly influence accelerated atherosclerosis development. Int J Cardiol (2013) 168:1965-74. doi: 10.1016/j.ijcard.2012.12.085

56. Goronzy JJ, Weyand CM. T-cell co-stimulatory pathways in autoimmunity. Arthritis Res Ther (2008) 10:1-10. doi: 10.1186/ar2414

57. Coussens LM, Werb ZJN. Inflammation and cancer. Nature (2002) 420:860-7. doi: 10.1038/nature01322

58. Egen JG, Kuhns MS, Allison JP. CTLA-4: new insights into its biological function and use in tumor immunotherapy. Nat Immunol (2002) 3:611-8. doi: 10.1038/ni0702-611

59. Schneider H, Downey J, Smith A, Zinselmeyer BH, Rush C, Brewer JM, et al. Reversal of the TCR stop signal by CTLA-4. Science (2006) 313:1972-5. doi: $10.1126 /$ science. 1131078

60. Picarda E, Ohaegbulam KC, Zang XJ. Molecular pathways: targeting B7-H3 (CD276) for human cancer immunotherapy. Clin Cancer Res (2016) 22:342531. doi: 10.1158/1078-0432.CCR-15-2428
61. Lim S, Liu H, Da Silva LM, Arora R, Liu Z, Phillips JB, et al. Immunoregulatory protein $\mathrm{B} 7-\mathrm{H} 3$ reprograms glucose metabolism in cancer cells by ROS-mediated stabilization of HIF1 $\alpha$. Cancer Res (2016) 76:2231-42. doi: 10.1158/0008-5472.CAN-15-1538

62. Tekle C, Nygren MK, Chen YW, Dybsjord I, Nesland JM, Maelandsmo GM, et al. B7-H3 contributes to the metastatic capacity of melanoma cells by modulation of known metastasis-associated genes. Int J Cancer (2012) 130:2282-90. doi: 10.1002/ijc.26238

63. Kang F-B, Wang L, Jia H-C, Li D, Li HJ, Zhang YG, et al. B7-H3 promotes aggression and invasion of hepatocellular carcinoma by targeting epithelialto-mesenchymal transition via JAK2/STAT3/Slug signaling pathway. Cancer Cell Int (2015) 15:1-11. doi: 10.1186/s12935-015-0195-Z

64. Zhang J, Wang J, Marzese DM, Wang X, Yang Z, Li C, et al. B7H3 regulates differentiation and serves as a potential biomarker and theranostic target for human glioblastoma. Lab Invest (2019) 99:1117-29. doi: 10.1038/s41374-0190238-5

65. Li Y, Yang X, Yao P, Shen W, Wu Y, Ye Z, et al. B7-H3 increases the radioresistance of gastric cancer cells through regulating baseline levels of cell autophagy. Am J Transl Res (2019) 11(7):4438-49.

66. McDougall SR, Anderson AR, Chaplain MA. Mathematical modelling of dynamic adaptive tumour-induced angiogenesis: clinical implications and therapeutic targeting strategies. J Theor Biol (2006) 241:564-89. doi: 10.1016/ j.jtbi.2005.12.022

67. Ricci-Vitiani L, Pallini R, Biffoni M, Todaro M, Invernici G, Cenci T, et al. Tumour vascularization via endothelial differentiation of glioblastoma stemlike cells. Nature (2010) 468:824-8. doi: 10.1038/nature09557

68. Wang R, Chadalavada K, Wilshire J, Kowalik U, Hovinga KE, Geber A, et al. Glioblastoma stem-like cells give rise to tumour endothelium. Nature (2010) 468:829-33. doi: 10.1038/nature09624

69. Xie C, Liu D, Chen Q, Yang C, Wang B, Wu H. Soluble B7-H3 promotes the invasion and metastasis of pancreatic carcinoma cells through the TLR4/NFкB pathway. Sci Rep (2016) 6:1-9. doi: 10.1038/srep27528

70. Lai H, Sun Z, Yang J, Wu P, Guo Y, Sun J. B7-H3 modulates endothelial cell angiogenesis through the VEGF cytokine. Immunol Res (2019) 67:202-11. doi: 10.1007/s12026-019-09084-w

71. Ferrara N. VEGF and the quest for tumour angiogenesis factors. Nat Rev Cancer (2002) 2:795-803. doi: 10.1038/nrc909

72. Li G, Quan Y, Che F, Wang L. B7-H3 in tumors: friend or foe for tumor immunity? Cancer Chemother Pharmacol (2018) 81:245-53. doi: 10.1007/ s00280-017-3508-1

73. Castellanos JR, Purvis IJ, Labak CM, Guda MR, Tsung AJ, Velpula KK, et al. B7-H3 role in the immune landscape of cancer. Am J Clin Exp Immunol (2017) 6(4):66-75.

74. Lupu CM, Eisenbach C, Lupu AD, Kuefner MA, Hoyler B, Stremmel W, et al. Adenoviral B7-H3 therapy induces tumor specific immune responses and reduces secondary metastasis in a murine model of colon cancer. Oncol Rep (2007) 18:745-8. doi: 10.3892/or.18.3.745

75. Yang HY, Chu M, Zheng LW, Zwahlen RA, Luo J, Zou DH, et al. Transgenic B7-H3 therapy induces tumor-specific immune response in human oral squamous cell cancer: an in vitro study. Oral Surg Oral Med Oral Pathol Oral Radiol Endod (2008) 106:721-8. doi: 10.1016/ j.tripleo.2008.08.012

76. Ma L, Luo L, Qiao H, Dong X, Pan S, Jiang H, et al. Complete eradication of hepatocellular carcinomas by combined vasostatin gene therapy and B7H3mediated immunotherapy. J Hepatol (2007) 46:98-106. doi: 10.1016/ j.jhep.2006.07.031

77. Loo D, Alderson RF, Chen FZ, Huang L, Zhang W, Gorlatov S, et al. Development of an Fc-enhanced anti-B7-H3 monoclonal antibody with potent antitumor activity. Clin Cancer Res (2012) 18:3834-45. doi: 10.1158/ 1078-0432.CCR-12-0715

78. Fauci JM, Sabbatino F, Wang Y, Londoño-Joshi AI, Straughn JM Jr., Landen $\mathrm{CN}$, et al. Monoclonal antibody-based immunotherapy of ovarian cancer: Targeting ovarian cancer cells with the B7-H3-specific mAb 376.96. Gynecol Oncol (2014) 132:203-10. doi: 10.1016/j.ygyno.2013.10.038

79. Kendsersky NM, Lindsay JM, Kolb EA, Smith MA, Teicher BA, Erickson S, et al. The B7-H3-targeting antibody-drug conjugate m276-SL-PBD is potently effective against pediatric cancer preclinical solid tumor models. Clin Cancer Res (2021), clincanres.4221.2020. doi: 10.1158/1078-0432.CCR-20-4221 
80. Scribner JA, Brown JG, Son T, Chiechi M, Li P, Sharma S, et al. Preclinical Development of MGC018, a Duocarmycin-based Antibody-drug Conjugate Targeting B7-H3 for Solid Cancer. Mol Cancer Ther (2020) 19:2235-44. doi: 10.1158/1535-7163.MCT-20-0116

81. Du H, Hirabayashi K, Ahn S, Kren NP, Montgomery SA, Wang X, et al. Antitumor responses in the absence of toxicity in solid tumors by targeting B7-H3 via chimeric antigen receptor T cells. Cancer Cell (2019) 35:22137.e228. doi: 10.1016/j.ccell.2019.01.002

82. Majzner RG, Theruvath JL, Nellan A, Heitzeneder S, Cui Y, Mount CW, et al. CAR T cells targeting B7-H3, a pan-cancer antigen, demonstrate potent preclinical activity against pediatric solid tumors and brain tumors. Clin Cancer Res (2019) 25:2560-74. doi: 10.1158/1078-0432.CCR-18-0432

83. Theruvath J, Sotillo E, Mount CW, Graef CM, Delaidelli A, Heitzeneder S, et al. Locoregionally administered B7-H3-targeted CAR T cells for treatment of atypical teratoid/rhabdoid tumors. Nat Med (2020) 26:712-9. doi: 10.1038/ s41591-020-0821-8

Conflict of Interest: The authors declare that the research was conducted in the absence of any commercial or financial relationships that could be construed as a potential conflict of interest.

Copyright (C 2021 Liu, Liang, Liu, Zhang, Wang, Watson, Zhou, Zhang, Wu, Zhang, Lu and Wang. This is an open-access article distributed under the terms of the Creative Commons Attribution License (CC BY). The use, distribution or reproduction in other forums is permitted, provided the original author(s) and the copyright owner(s) are credited and that the original publication in this journal is cited, in accordance with accepted academic practice. No use, distribution or reproduction is permitted which does not comply with these terms. 